\title{
Aging enhances cognitive biases to friends but not the self
}

\author{
Jie Sui ${ }^{1}$ • Glyn W. Humphreys ${ }^{2}$
}

Published online: 17 March 2017

(C) The Author(s) 2017. This article is published with open access at SpringerLink.com

\begin{abstract}
We measured changes in self and friend biases in perceptual matching in young and older participants. Participants learned associations between neutral geometric shapes and three personal labels (You, Friend, or Stranger), representing themselves, their named best friend, and a stranger not corresponding to anyone they knew. They then responded whether the shapes and labels matched or mismatched. In addition, participants reported the perceived personal distance between themselves, their best friend, and a stranger. Relative to young participants, older adults showed an increased bias toward matching their friends over strangers, whereas the bias toward the self over friends tended to decrease. Equivalent results occurred for a perceived personal distance measure, and, on measures of perceptual sensitivity with older participants, the personal distance between friends and strangers correlated with the friend bias in matching. These results indicate that the social bias toward a familiar best friend increases with age and modulates perceptual matching.
\end{abstract}

Keywords Aging - Social perception - Distance perception . Self-control $\cdot$ Response bias

Considerable work has shown changes in cognition as people age. Most typically, there are reductions in memory,

Jie Sui

j.sui@bath.ac.uk

1 Department of Psychology, University of Bath, Bath BA2 7AY, UK

2 Department of Experimental Psychology, University of Oxford, Oxford OX1 3UD, UK executive functions, and processing speed in older populations (Grady \& Craik, 2000; Rabbitt, 1997; Salthouse, 2009), though more "crystallized" functions such as language tend to be preserved (Rabbitt, 1997). Changes in social cognitive functions as people age have been studied less extensively. For example, there are reports that older people have more difficulty seeing other people's perspective in "theory of mind" (ToM) tasks (Slessor, Phillips, \& Bull, 2007, 2008). Older adults are also more affected by selfrelated stimuli than younger participants, when asked to memorize material (Mather \& Carstensen, 2005; Trelle, Henson, \& Simons, 2015), consistent with lay reports that older people become more self-absorbed and inwardlooking. These enhancing effects of self bias are found even when comparisons are made under conditions in which the stimuli are semantically encoded (Trelle et al., 2015), suggesting that the effects do not simply reflect the depth of processing. The reasons for these changes are unclear, however. For example, performance in ToM tasks is known to be influenced by executive capacity (Apperly, Samson, Chiavarino, \& Humphreys, 2004), so the reduced ToM performance in older adults may reflect less executive control (Cavallini et al., 2015; Gutchess, Kensinger, \& Schacter, 2010; Henry, Phillips, Ruffman, \& Bailey, 2013; ShamayTsoory \& Aharon-Peretz, 2007). Alternatively, problems on ToM tasks may be due to the increased saliency of selfrelated knowledge, which is often pitted against the information held by the other person in ToM tasks (e.g., in the Sally-Anne task, in which the observer has knowledge of where an object has been moved to, after a stooge has walked out of a room; cf. Wimmer \& Perner, 1983). It is also unclear whether increased influences of self-related information in memory reflect a strategic emphasis under conditions of reduced memory capacity, or whether this reflects changes in self-related processing more generally. 
We set out to address these questions in a study on the effects of aging on self-related processing and on the processing of other people, in a simple perceptual-matching task that has previously been shown to produce highly reliable biases favoring selfrelated stimuli (Sui, He, \& Humphreys, 2012) that are stable across individuals over time (Humphreys \& Sui, 2015; Stolte, Humphreys, Yankouskaya, \& Sui, 2015). We had young ( $<30$ years) and two groups of older adults (60-69 and 69+ years) ${ }^{1}$ make associations between personal labels (You, Friend, and Stranger) and neutral geometric shapes (square, circle, and triangle), and they were told that the labels referred to themselves, their best friend, or a stranger who was not anyone they knew. Subsequently, participants either saw the original shape-label pairs (e.g., square-you, circle-friend) or saw the stimuli repaired (e.g., square-friend, circle-you). The task was to decide whether the stimuli were in their original pairing or had been repaired. Young participants show a large advantage for matching self-related pairs (square-you) over stimuli that they associate with their best friend (circle-friend), as well as an advantage for friend-related stimuli over items associated with a stranger (triangle-stranger; Sui et al., 2012). This self advantage increases when stimuli are perceptually degraded, suggesting enhanced perception for self-related stimuli. We asked whether this basic form of self bias in the perceptual-matching task is modulated by age.

Previous work has consistently shown that memory in young adults is better for self-related stimuli than for stimuli related to other people - the self-reference effect in memory (Conway \& Pleydell-Pearce, 2000; Cunningham, Turk, Macdonald, \& Macrae, 2008; Symons \& Johnson, 1997). Recent research has shown that this self bias in memory is maintained in older people, though they have reduced memory capacity in general (e.g., Gutchess Kensinger, \& Schacter, 2010; Gutchess, Kensinger, Yoon, \& Schacter, 2007). Contrasting to past work on memory, in the present study we tested the effect of aging on the perceptual-matching task. One interesting aspect of the self-bias effect in perceptual matching is that it is related to an underlying neural circuitry (Sui, Rotshtein, \& Humphreys, 2013). Notably, activation of ventral brain regions is associated with self processing and attention (respectively in the ventro-medial prefrontal cortex [VMPFC] and the posterior superior temporal sulcus) when self-related stimuli are presented, whereas enhanced activation of a dorsal frontoparietal network is associated with attentional control when participants respond to stimuli related to a stranger. These data indicate that attentional-control processes are recruited more strongly in the latter condition (see Sui \& Humphreys, 2015a, for a discussion). Additionally, neuropsychological studies have shown that

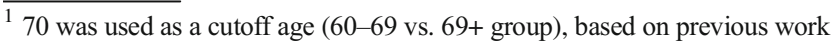
with a large sample suggesting that 70 may be the critical period for the determinants of cognitive aging (Avlund, Kreiner, \& Schultz-Larsen, 1993; Gow \& Mortensen, 2016).
}

brain lesion over the VMPFC abolishes self biases in memory, whereas brain damage in the dorsal attentional-control network that spares the VMPFC generates abnormally large biases toward self-related stimuli, due to an exaggerated effect of strong attentional signals (Sui, Enock, Ralph, \& Humphreys, 2015). Researchers have argued that self-bias effects are determined by the interaction between the ventral network through the VMPFC and the dorsal attentional-control network for control of behavior (Sui, 2016). From these findings, we may predict that self biases in perceptual matching may increase as people age because of a reduction in executive control, which should most strongly affect responses to stimuli associated with strangers.

On the other hand, work from the domain of social and clinical psychology has suggested that changes in cognition in older adults can reflect strategic changes in processing. For example, older adults appear to place more attentional weight on positive relative to negative information, leading to stronger positivity biases in memory than in younger participants (Mather \& Carstensen, 2005). Older adults also show biases in social preference toward a smaller number of familiar social partners, partly because of strategic reflection on the lack of perceived time, as well as greater response to the positive emotional associations linked to such partners (Fung, Carstensen, \& Lutz, 1999). These results offer alternative hypotheses about how older adults may vary in their perceptual matching for self, friend, and stranger-related stimuli. For example, if aging leads to an increased bias toward friend-related stimuli, due to the weighting of positive emotion and familiarity, it is possible that the friend advantage (relative to performance with stimuli related to a stranger) may increase as people age, whereas the self advantage (relative to the friend) may actually decrease. This would contradict an account based on the effects of decreasing executive function in older adults for the more difficult associations (friend and stranger).

In summary, we assessed the effects of aging on selfand other-related processing in the perceptual-matching task. We would predict an enhancing effect of self biases as people age, if reduced executive capacity affects responses to other-related stimuli in perceptual matching. Alternatively if strategic changes in processing among older adults modulate perceptual matching by increasing the weight on positive emotions and familiarity linked to close others, then we might expect an increased effect to their best friend with a decreased bias to the self over friends. To provide additional evidence on the latter possibility, we not only had participants carry out perceptual matching, but also asked them to rate the perceived personal distances between themselves, their best friend, and a (named) stranger. If aging biases processing toward a 
familiar friend, the judged personal distances from the self to the friend and from the friend to a stranger might vary across different age groups (the self distance from the friend reducing and the friend distance from the stranger increasing). Moreover, variations in perceived personal distance may predict the self and friend biases on perceptual matching. This would provide converging evidence for an effect of social coding, rather than executive functions per se, modulating cognition in older adults.

\section{Method}

\section{Participants}

We recruited 103 participants in total, including 36 in the young group (nine males, 27 females; ages 19-29 years, mean age $22.08 \pm 2.60$ years), 33 participants in the 60 69 group (16 males, 17 females; ages 61-69 years, mean age $65.58 \pm 2.42$ years), and 34 participants (15 males, 19 females; ages $70-86$ years, mean age $75.59 \pm 4.69$ years) in the $69+$ group. The sample size was determined from prior studies in order to get a reasonable effect size (e.g., Humphreys \& Sui, 2015; Sui et al., 2012). All participants were right-handed and had normal or corrected-to-normal vision. Informed consent was obtained from all participants prior to the experiment according to procedures approved by the ethics committee of the Medical Sciences Division of Oxford University.

\section{Stimuli and tasks}

Three geometric shapes (triangle, circle, and square) were assigned to three personal labels representing the participant, his or her named best friend (whom he or she was asked to name), and a stranger (he or she was asked to give a name that was familiar but not held by any personal acquaintance). The assignment of shapes to the different people was counterbalanced across participants. Before the experiment, participants were asked to name one of their gender-matched best friends and to select a gender-matched name as a stranger from a list of common names for people they did not know personally. The experiment then began with a shape-person association instruction presented on the screen. For example, participants were told that "the triangle represents your best friend, Mary, the square represents yourself, and the circle represents a stranger, Lucy." The order of the three shape-label assignments during the instruction was counterbalanced across participants. The shape images were not presented at this stage. The instruction took about $1 \mathrm{~min}^{2}$

\footnotetext{
${ }^{2}$ Previous studies have consistently shown that participants can immediately learn shape-label associations after the instruction (e.g., Stolte et al., 2015; Sui et al., 2012).
}

After the associative instruction, participants immediately performed a shape-label matching task to judge whether a shapelabel pair matched. The same three shapes were used throughout all of the experimental trials. A shape occupying $3.5^{\circ} \times 3.5^{\circ}$ of visual angle appeared above a white central fixation cross, $0.8^{\circ} \times$ $0.8^{\circ}$ of visual angle. One of three personal labels (You, Friend, or Stranger, covering $1.76^{\circ} / 2.52^{\circ} \times 1.76^{\circ}$ of visual angle) was displayed below the fixation cross. The task was to decide whether the shape-label pair was the same one initially shown or whether the shape and label had been re-paired. All stimuli were displayed on a gray background. The E-Prime software (version 2.0) was used to present the stimuli and record responses. The experiment was run on a PC with a 22 -in. monitor $(1,920 \times 1,080$ pixels) at $60 \mathrm{~Hz}$.

Following the matching task, participants were required to indicate the personal distance between any two people by making two marks on a straight line on an A4 sheet (i.e., self and stranger, friend and stranger, self and friend), with the physical distance between the marks (in millimeters) serving as an index of the personal distance between the individuals (see Fig. $2 b$ in the Results, as well as Sui \& Humphreys, 2015b). The friend and the stranger referred to those whom participants had named before the experiment. There were ten trials per pairing; half the trials started with one person (e.g., self-friend), and the other half started with the other person (friend-self). To rule out individual variations across the age groups, personal distance (i.e., self-friend and friend-stranger) was also scaled by the distance between the participant and the stranger as relative scores (see Fig. 3 below). One participant in the 60-69 group and two participants in the 69+ group did not report personal distance.

\section{Procedure}

Participants completed the experiment individually in a quiet testing room. For the matching task, participants were instructed to associate one of three shapes with themselves, their best friend, or a stranger. No images of stimuli were displayed during the instruction stage (e.g., "circle = you," "square = your best friend," "triangle = a stranger"). After the instruction, participants had to judge whether a simultaneously presented shape-and-label pair matched (Fig. 1). Each trial started with a central fixation cross for $500 \mathrm{~ms}$, followed by a shape-label pair at the center of the screen, for $100 \mathrm{~ms}$ for the young group and $500 \mathrm{~ms}$ for the older participants. Half of the pairings of the shape and label conformed to the instruction and were responded to as match trials; on the remaining trials, the shapes and labels were re-paired to form mismatch trials. For mismatch trials, a shape was paired with each of the other two possible labels (e.g., the self shape with either the friend or the stranger label). The next frame was a blank field lasting 
$1,000 \mathrm{~ms}$ for the young participants, or 3,000 $\mathrm{ms}$ for the old participants. ${ }^{3}$ Participants were encouraged to make a response as quickly and accurately as possible within this interval by pressing one of the two keys on a keyboard with the index or middle finger of the right hand. The order of the response keys was counterbalanced across participants. A feedback message (Correct, Incorrect, or Too Slow!) was then given at the center of the screen for $500 \mathrm{~ms}$. Participants were also informed of their overall accuracy at the end of each block. The experiment consisted of six blocks of 60 trials each, following 24 practice trials. ${ }^{4}$ Thus, there were 60 trials for each match and mismatch condition.

\section{Data analysis}

Raw scores (Fig. 1) For the behavioral measures, there was no trade-off between reaction times (RTs) and accuracy for any condition. The data on RTs and the $d$-prime results (computed from performance on match trials contrasted against mismatch trials with the same shape; see Sui et al., 2012) are reported separately. The data analyses for RTs were conducted separately for the match and mismatch trials, due to the different responses being made in these cases (e.g., Sui \& Humphreys, 2014; Sun, Fuentes, Humphreys, $\&$ Sui, 2016). Repeated measures analyses of variance (ANOVAs) were conducted with Association (self, friend, or stranger) as a within-subjects factor and Age (young, 6069 , or 69+) as a between-subjects factor. For the measures of self-reported personal distance, we conducted repeated measures ANOVAs with Distance (self-friend, self-stranger, or friend-stranger) as a within-subjects factor and Age (young, 60-69, or 69+) as a between-subjects factor.

Bias scores To assess changes in the self and friend biases over age, as well as to rule out individual variation across the three age groups, both the behavioral and self-reported data were normalized. We conducted ANOVAs using normalized bias scores with Bias (self vs. friend) as a withinsubjects factor and Age (young, 60-69, or 69+) as a between-subjects factor. For RTs, the self bias was measured by the difference in performance for the self versus the friend condition, divided by the sum across the two conditions. The friend bias was indexed by the difference in performance for the friend condition against the stranger condition, divided by the sum across the two conditions.

\footnotetext{
${ }^{3}$ The exposure durations and blank intervals differed across the age groups in order to achieve comparable accuracies of the matching associations, which were tested in a pilot session.

${ }^{4}$ If older participants failed to complete the 24 practice trials, stimuli were then presented on the screen until a response was made. In all, five out of the 34 in the 69+ group and one out of the 33 in the 60-69 group made self-paced responses. The accuracy data for these participants fell within the range of the participants with a fixed duration, and therefore the data from all participants in each group were collapsed.
}

The self bias was indexed by the differential scores relative to the friend rather than the stranger condition so that we could compare the differences between the self and friend biases while controlling the familiarity effect. For $d^{\prime}$, the self and friend biases were indexed by the differential scores between the two conditions. For both RTs and $d^{\prime}$, a larger score indicated a larger bias, whereas smaller scores indicated a relatively a small bias. To normalize self-reported personal distance, we computed the selffriend distance scaled by the ratio of the participant-best friend distance to the self-stranger distance; the friendstranger distance was measured by the ratio of the best friend-stranger distance to the self-stranger distance. In this case, a larger score indicated a greater relative personal distance between the two people, whereas a smaller score indicates a relatively shorter personal distance. There were similar patterns in RTs and in self-reported personal distance (Fig. 2).

These bias scores were also used to calculate the relations between the behavioral measures and the self-reported personal distance scores. We conducted Spearman correlation analyses between the two types of measures. Given the ANOVA results indicating differences between the young and older participants, correlations were calculated for young and older participants separately.

For all critical comparisons, Holm-Bonferroni corrections at $\alpha=.05$ were applied to all multiple comparisons (Holm, 1979). We report effect sizes using partial eta-squared $\left(\eta_{\mathrm{p}}{ }^{2}\right)$ for ANOVAs and Cohen's $d z$ for $t$ tests (Lakens, 2013).

\section{Results}

\section{Behavioral measures}

Match and mismatch trials were analyzed separately, since they reflect separate decision criteria (see Sui et al., 2012).

RTs in match trials: Absolute RTs The analysis of match trials revealed a significant main effect of association, $F(2$, $200)=44.62, p<.001, \eta_{\mathrm{p}}{ }^{2}=.31$, with faster responses to the self than to friend-associated $(p=.001)$ and strangerassociated $(p<.001)$ trials, and faster responses to the friend than to stranger-associated trials $(p<.001)$. We also discovered a significant main effect of age, $F(2,100)=$ 22.38, $p<.001, \eta_{\mathrm{p}}{ }^{2}=.31$, reflecting faster responses in the young than in the $60-69(p<.001)$ and $69+(p<.001)$ groups, as well as faster responses in the 60-69 than in the $69+$ group $(p=.03)$. The main effects were qualified by a significant interaction between the association and age, $F(4,200)=3.99, p<.005, \eta_{\mathrm{p}}{ }^{2}=.07$ (Fig. 2a). The interaction was broken down for each of the three groups separately. The analysis in the young group showed a 


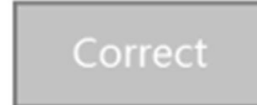

or

Incorrect

Feedback (500 ms)

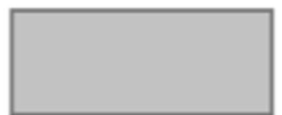

Blank (1000/3000 ms)

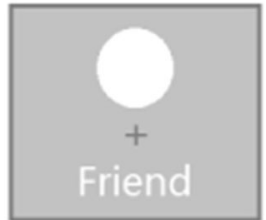

Match $(100 / 500 \mathrm{~ms})$ or

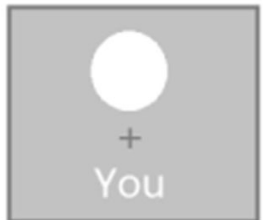

Mismatch (100/500 ms)

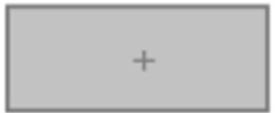

Fixation $(500 \mathrm{~ms})$

Fig. 1 Examples of the stimuli and trial procedure in the matching task. A trial began with a 500-ms fixation displayed in the center of the screen, followed by a shape-label pairing presented for $100 \mathrm{~ms}$ for young participants or $500 \mathrm{~ms}$ for older individuals. The response deadlines were $1,000 \mathrm{~ms}$ for young participants or $3,000 \mathrm{~ms}$ for older adults. A $500-\mathrm{ms}$ feedback presentation was then given to indicate whether a response was correct significant effect of association, $F(2,70)=60.44, p<$ $.001, \eta_{\mathrm{p}}^{2}=.63$, with faster responses to the self than to both friend-associated $[t(35)=-8.62, p<.001, d z=$ $1.44]$ and stranger-associated $[t(35)=-8.32, p<.001$, $d z=1.37]$ trials, and faster responses to the friend than to stranger trials $[t(35)=-2.79, p=.008, d z=0.44]$. We also found a significant effect of association in the 60-69 group, $F(2,64)=20.32, p<.001, \eta_{\mathrm{p}}{ }^{2}=.39$. Unlike in the young group, no difference in responses emerged between the self and friend trials $[t(32)=$ $1.25, p=.22, d z=0.22]$, but there were faster responses to both the self $[t(32)=-6.92, p<.001, d z=1.21]$ and friend $[t(32)=-4.19, p=.001, d z=0.73]$ trials than to the stranger trials. Likewise, a significant effect of association was apparent in the $69+$ group, $F(2,66)=11.99$, $p<.001, \eta_{\mathrm{p}}^{2}=.27$, reflecting faster responses to the self $[t(33)=-3.88, p<.001, d z=0.66]$ and friend $[t(33)=-3.85, p=.001, d z=0.66]$ trials than to the stranger trials, but we observed no difference in responses to the self and friend trials $[t(33)=-1.85, p=$ $.07, d z=0.32]$.

Bias measures Each of the biases was assessed across the three age groups separately using normalized bias scores, with Bias (self vs. friend; see the Method section) as a

b
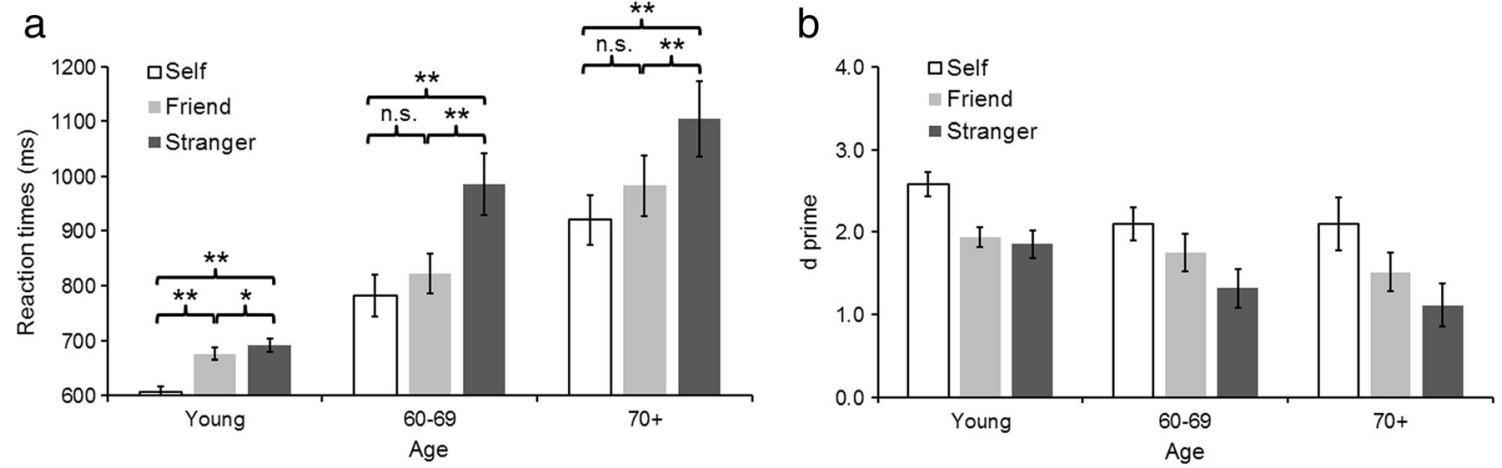

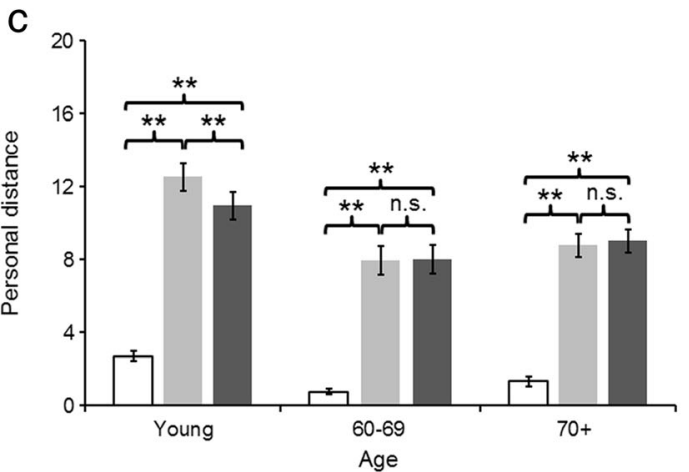

Fig. 2 (a, b) Performance in perceptual matching as a function of age (young, 60-69, or 69+) and association (self, friend, or stranger), shown for absolute reaction times (a) and $d^{\prime}(\mathbf{b})$. (c) Absolute personal distance measures as a function of age (young, 60-69, or 69+) and comparison (self-friend, self-stranger, or friend-stranger). Error bars represent one standard error 
within-subjects factor and Age (young, 60-69, or 69+) as a between-subjects factor. The normalized scores here equated to differences in baseline RTs across the age groups. The analysis failed to show a significant main effect of either bias or age, $F(2,100)=1.14, p=.29 ; F(2,100)=2.08, p=$ .13. However, we did observe a significant interaction between bias and age, $F(2,100)=6.20, p=.003, \eta_{\mathrm{p}}{ }^{2}=.11$. The between-subjects analysis failed to show significant differences across the age groups in the self-bias effect, $F(2,100)=1.42, p=.25$ (Fig. 3, upper left figure). However, there was a significant effect of age for the friend bias, $F(2,100)=9.64, p<.001, \eta_{\mathrm{p}}{ }^{2}=.16$. Both the 60-69 $[t(67)=4.70, p<.001, d z=1.13]$ and $69+[t(68)=3.06, p=$ $.003, d z=0.73]$ groups showed larger friend biases than did the young group, but we found no difference between the 60-69 and 69+ groups $[t(65)=1.49, p=.14, d z=0.36]$ (Fig. 3, upper right figure). These results indicated an enhanced friend bias with age.

To verify this result, we compared how many participants showed a larger self than friend bias within the young versus the old (60-69 and 69+) groups. Relative to the older participants (combining the 60-69 and 69+ age groups), young participants were more likely to demonstrate a larger self bias than a friend bias $(83 \%$ of individuals in the young group vs. $30 \%$ of individuals in the old groups), $\chi^{2}=$ $26.82, p<.001$.

RTs on mismatch trials The analysis of mismatch trials did not show either a significant main effect of association, $F(2$, 200) $=2.52, p=.08, \eta_{\mathrm{p}}{ }^{2}=.03$, or an interaction between association and age, $F(4,200)=0.85, p=.49, \eta_{\mathrm{p}}{ }^{2}=.02$. However, a significant main effect of age, $F(2,200)=$ 21.23, $p<.001, \eta_{\mathrm{p}}{ }^{2}=.30$, reflected faster responses in the young than in either the $60-69(p<.001)$ or the $69+(p<.001)$ group. No differences emerged between the 60-69 and 69+ groups $(p=.46)$.

d-prime The analysis of $d$ ' showed a significant main effect of association, $F(2,200)=56.20, p<.001, \eta_{\mathrm{p}}{ }^{2}=.36$, with larger $d^{\prime}$ scores for the self condition than for the friend $(p<.001)$ and stranger $(p<.001)$ conditions, and a greater $d^{\prime}$ in the friend than in the stranger condition $(p<.001$; Fig. 2b). However, neither the main effect of age nor the interaction between association and age was significant, $F(2,100)=2.06, p=$ $.13, F(4,200)=1.46, p=.22$.

In line with the RT data, we also conducted a chi-square analysis for the numbers of participants with a large or a small self bias relative to their friend bias. The analysis

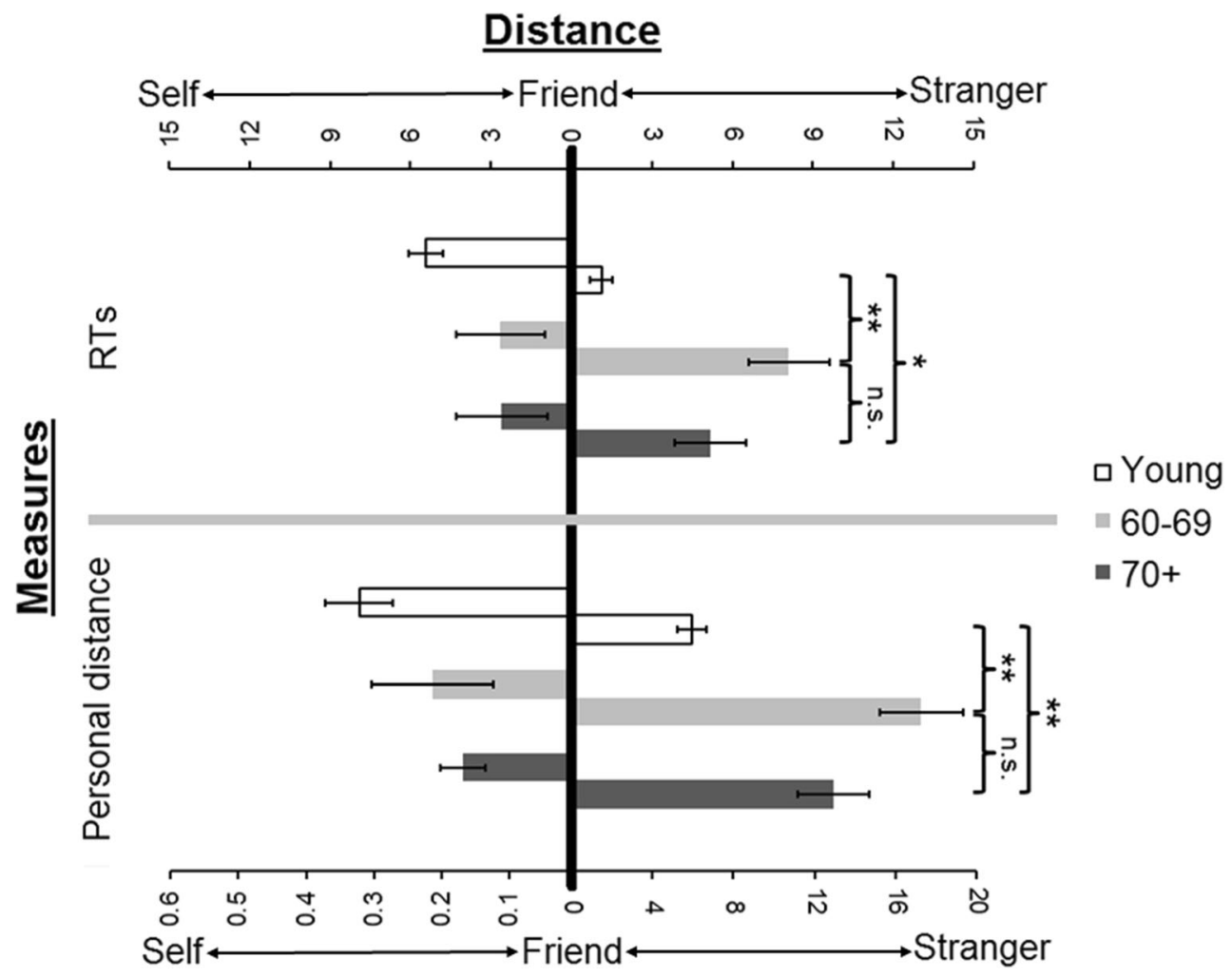

Fig. 3 Patterns of self (vs. friend) and friend (vs. stranger) biases in reaction times (RTs, top panel) and self-reported personal distance (bottom panel). The plots on the left side represent self biases in RTs (upper part) and in self-reported personal distance (lower part). The

plots on the right side represent friend biases in RTs (upper part) and self-reported personal distance (lower part). Error bars represent one standard error of the normalized differential scores 
showed that the participants in the young group were more likely to have a large self bias (vs. friend bias; $81 \%$ of individuals in the young group) than were those in the old (6069 and $69+$ ) groups (55\% of individuals in those groups), $\chi^{2}$ $=6.53, p=.01$.

\section{Personal distance}

Absolute distance The analysis demonstrated a significant main effect of distance, $F(2,194)=371.13, p<.001, \eta_{\mathrm{p}}{ }^{2}=$ .79; the rated distance was shorter for the self-friend comparison than for the self-stranger $(p=.001)$ and friendstranger $(p<.001)$ comparisons, and the rated distance for friend-stranger was shorter than that for self-stranger $(p=.046)$. We also observed a significant main effect of age, $F(2,97)=9.49, p<.001, \eta_{\mathrm{p}}{ }^{2}=.16$; young individuals reported larger personal distances than did the 60-69 $(p<$ $.001)$ and $69+(p=.015)$ groups, but there was no difference between the 60-69 than 69+ groups $(p=.57)$. The interaction between distance and age was marginal, $F(4$, 194) $=2.22, p=.068, \eta_{\mathrm{p}}^{2}=.07$ (Fig. 2c).

In line with the RT analysis, this interaction was broken down into the three age groups. The young group showed a significant effect of distance, $F(2,70)=124.57, p<.001, \eta_{\mathrm{p}}{ }^{2}=$ .78 , with a larger distance in the self-stranger than in the selffriend $[t(35)=13.32, p<.001, d z=2.21]$ and friend-stranger $[t(35)=4.32, p<.001, d z=0.25]$ comparisons, and the distance was larger for the friend-stranger than for the self-friend comparison $[t(35)=10.14, p<.001, d z=2.56]$. The effect of distance was also significant in the 60-69 group, $F(2,62)=$ $102.37, p<.001, \eta_{\mathrm{p}}^{2}=.77$. Unlike in the young group, no difference in distance was apparent between self-stranger and friend-stranger $[t(31)=-0.33, p=.74, d z=0.07]$, but the distances were both larger for the self-stranger $[t(31)=$ 10.28, $p<.001, d z=3.04]$ and friend-stranger $[t(31)=$ 10.27, $p<.001, d z=3.47]$ comparisons than for the selffriend comparison. Similarly, there was a significant effect of distance in the 69+ group, $F(2,62)=175.24, p<.001$, $\eta_{\mathrm{p}}{ }^{2}=.85$. The distances were comparable for the self-stranger and friend-stranger comparisons $[t(31)=-1.31, p=.27, d z=$ $0.01]$, whereas there was a larger rated distance for the selfstranger $[t(31)=13.60, p<.001, d z=2.21]$ and friend-strang$\operatorname{er}[t(31)=14.05, p<.001, d z=2.19]$ comparisons than for the self-friend comparison.

Bias scores To assess the aging effect on perceived personal distance, we conducted ANOVAs using relative distance scores, normalized by the rated distance between the self and the stranger (to equate for possible age differences in the lengths of the scales used). The Bias in distance was a within-subjects factor (self-friend vs. friend-stranger; see the Method section), and Age was a between-subjects factor (young, 60-69, or 69+). We found a significant main effect of distance, $F(2,97)=167.35, p<.001, \eta_{\mathrm{p}}{ }^{2}=.63$, reflecting a smaller distance in the self-friend than in the friend-stranger comparison. The effect of age was also significant, $F(2,97)=14.06, p<.001, \eta_{\mathrm{p}}{ }^{2}=.23$, with larger relative perceived personal distances in both the 60-69 $(p<.001)$ and $69+(p=.006)$ groups than in the young group, but no difference between the 60-69 and $69+$ groups $(p=.15)$. We also observed a significant interaction between personal distance bias and age, $F(2,97)=$ $13.65, p<.001, \eta_{\mathrm{p}}^{2}=.22$.

For the self-friend distance (the self-bias effect), there was no significant effect of age, $F(2,97)=1.84, p=.17$ (Fig. 3, the lower left figure). In contrast, we did find a significant effect of age for the friend-stranger distance (the friend-bias effect), $F(2,97)=13.86, p<.001, \eta_{\mathrm{p}}{ }^{2}=$ .22: Older participants in both the 60-69 $[t(66)=5.46, p<$ $.001, d z=1.33]$ and $69+[t(66)=3.85, p<.001, d z=0.94]$ groups assigned a greater distance between their best friend and the stranger than did the young participants [we found no difference between the 60-69 and 69+ groups; $t(62)=1.620, p=.11, d z=0.041]$ (Fig. 3, lower right figure). These results indicated an enhanced perceived friend-stranger distance, whereas the self-friend distance was maintained over age.

There were similar patterns in both RTs and self-reported personal distance (Fig. 3). Next, we conducted correlation analyses to assess the relations between these two types of measures.

\section{Correlations between behavioral measures and personal distance}

We conducted correlation analyses for young and older (6069 and 69+) participants separately. We calculated correlations between the friend and self biases in normalized behavioral scores (for both friend-stranger and self-friend) and personal distance (friend-stranger/self-stranger and selffriend/self-stranger) (see the Method section). For $d^{\prime}$, Spearman correlation analyses in the young participants failed to show any indication of significant correlations between the behavioral biases and perceived personal distance, $r \mathrm{~s}=-.01$ and $-.06, p \mathrm{~s}=.96$ and .73 , for the friend and self biases, respectively (Fig. 4, upper panel). In contrast, for the older group, we observed a significant positive correlation between the behavioral friend bias and perceived friendstranger distance, $r=.36, p=.003$ (Fig. 4, lower left plot); older adults who tended to show a larger reported personal distance between friends and strangers also showed greater increases in perceptual sensitivity in matching friend- over stranger-associated shapes. However, no significant correlation was apparent in self biases, $r=.05, p=.72$ (Fig. 4, lower right panel). For RTs, no significant correlations appeared for either the young or the older groups, $p \mathrm{~s}>.36$ 
a Friend-bias in young group

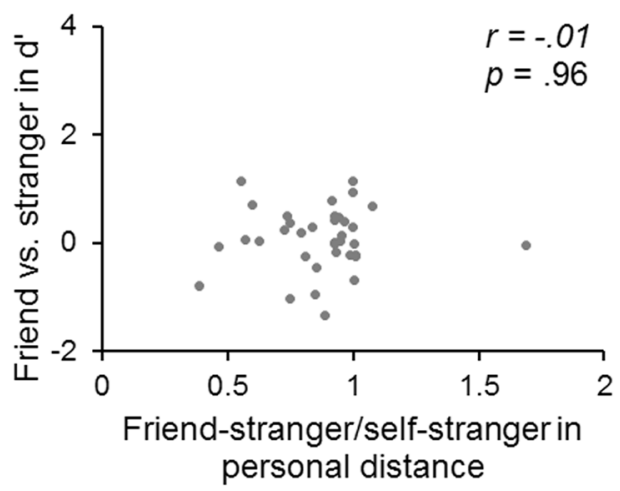

b Friend-bias in elderly group

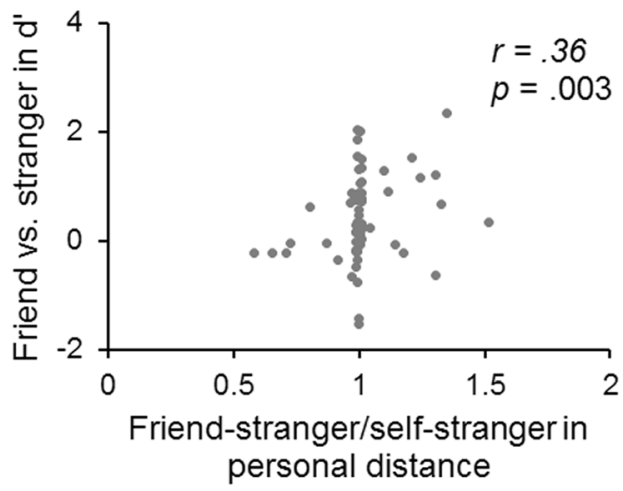

Fig. 4 Spearman correlations between normalized differential scores in $d^{\prime}$ and self-reported personal distance. (a) For the young group, no significant correlations are apparent in either friend bias (upper left plot)

\section{Discussion}

The results showed that self-bias effects on perceptual matching were at least maintained across the age range (when bias effects were normalized for absolute RTs; but in terms of absolute RTs, self biases over familiar friends decreased with age). These data on perceptual matching were paralleled by the findings when participants rated the personal distance between themselves, their familiar friend, and a named stranger. The perceived distance between the self and the friend was maintained across the age groups (Fig. 3). This runs counter to the view that exacerbated executive demands on older participants reduce matching performance on other-related stimuli, and thus increase the self-bias effect (cf. Sui \& Humphreys, 2015a), but these findings in perceptual matching are consistent with prior studies of the self-referential effect on memory (Gutchess et al., 2010; Gutchess et al., 2007), suggesting maintained effects of self-reference at different stages of processing more generally.

On the other hand, older participants showed larger friend biases than did younger participants (against a baseline of responses to strangers). This result in terms of the friend bias is consistent with the view that, relative to younger participants, older individuals tend to weigh familiar friends more in their
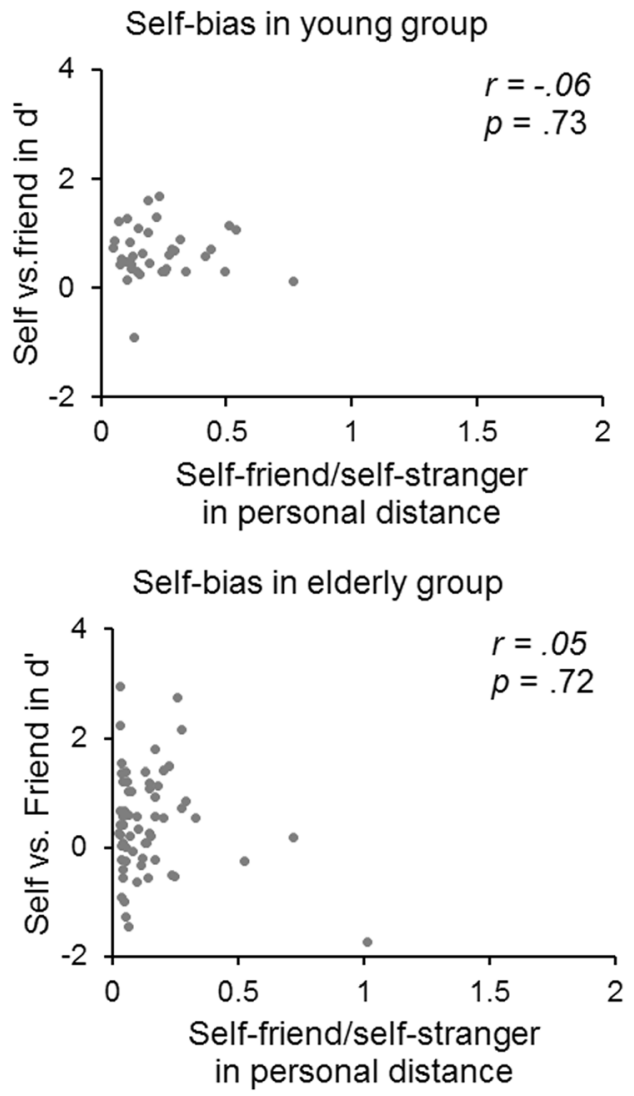

or self bias (upper right plot). (b) For the older group, a significant positive correlation can be seen in friend bias (lower left plot), but not in self bias (lower right plot)

perceptual judgments. It may also reflect an enhanced familiarity of their friends. Older participants are likely to have known their best friend for longer than younger participants, and the strength of the response to the friend may depend on the familiarity of the person. A further possibility (not mutually exclusive) is that older participants may show a more positive emotional response to their best friend partly because older individuals weight positive emotional value higher than do younger participants (Fung et al., 1999; Mather \& Carstensen, 2005). A positive emotional response to the friend association may enhance the friend bias (see Stolte et al., 2015). Consistent with these results reflecting the social coding of friend relative to stranger stimuli, we found that the rated personal distance of the best friend from the stranger increased with age. Additionally, we observed a correlation between the friend bias (friend-stranger) in the $d^{\prime}$ measure in perceptual matching and the rated perceptual distance between the friend and the stranger: Individuals who rated the friend as being more distant from the stranger also showed a larger bias in their sensitivity when matching friend over stranger stimuli in perceptual matching. Aron and colleagues proposed that individuals use close relationships to achieve self-expansion through the inclusion of others in the self (Aron, Aron, \& Norman, 2001; Aron, Aron, $\&$ Smollan, 1992). The present results are in line with the view 
that older participants favor their best friend more than do young adults. The increased inclusion of the friend in the self in older adults, then, may lead to a larger rated personal distance between the friend and the stranger, and a larger bias toward the friend over stranger stimuli in the matching task, but this was contradicted by the maintained self bias (vs. the friend) in perceptual matching, although the bias toward the self over friends did tend to decrease with age (see Fig. 3, upper left plot). We found no other direct relationships between perceived personal distance and biases in perceptual matching. We conclude that both the rating of personal distance and the sensitivity in perceptual matching are modulated by the social coding of the friend relative to the stranger, which changes as people age. In contrast, biases toward the self in perceptual matching do not increase with age.

A somewhat different account of the changes in self- and friend-related processing in older people is based on interference effects. ${ }^{5}$ Because it has been shown that older adults are more susceptible to the effects of proactive interference (Bowles \& Salthouse, 2003; Emery, Hale, \& Myerson, 2008), their maintained self bias and enhanced friend bias may stem from an increased proactive interference across trials in older people. For example, built-up interference may be stronger from self- and friend-related trials (trial $n-1$ ) to stranger-related trials (trial $n$ ) than is the interference from stranger-related trials (trial $n-1$ ) to self- and friend-related trials $(\operatorname{trial} n)$. However, the design of the present study did not allow for such analyses. These ideas may be tested in the future by formal analyses of carryover effects.

Though there are reductions in social-cognitive capacities (e.g., seeing other people's perspectives in ToM tasks) as well as in cognitive functions (e.g., memory and executive control functions) in older people, the present results confirm that the basic form of self bias in perceptual matching is preserved as people age. This is consistent with evidence from previous work in the memory domain (e.g., recall, recognition, and source memory) showing a maintained self advantage in memory among older participants (Gutchess et al., 2010; Gutchess et al., 2007; Hamami, Serbun, \& Gutchess, 2011; Mueller, Wonderlich, \& Dugan, 1986; Rosa \& Gutchess, 2011; Yang, Truong, Fuss, \& Bislimovic, 2012). Importantly, we also found an enhancing effect of the friend bias in perceptual matching, which correlated with the perceived personal distance between friends and strangers. These results have broad implications for understanding social behavior in older people. For example, they can help explain why older adults become more selective toward familiar friends when asked to make social judgments (Fredrickson \& Carstensen, 1990), since cognitive biases toward familiar friends would be reinforced by enhanced perception and attention to these individuals and to the information associated with them.

\footnotetext{
${ }^{5}$ We thank an anonymous referee for this comment.
}

Author note This article is dedicated to the memory of G.W.H. (19542016). This work was supported by grants from a European Research Council Advanced Investigator award (Pepe: 323883) (WT 106164MA) to G.W.H., the National Science Foundation (31371017) to J.S., and to both authors from the Economic and Social Research Council (ES/K013424/1).

Open Access This article is distributed under the terms of the Creative Commons Attribution 4.0 International License (http:// creativecommons.org/licenses/by/4.0/), which permits unrestricted use, distribution, and reproduction in any medium, provided you give appropriate credit to the original author(s) and the source, provide a link to the Creative Commons license, and indicate if changes were made.

\section{References}

Apperly, I. A., Samson, D., Chiavarino, C., \& Humphreys, G. W. (2004). Frontal and temporo-parietal lobe contributions to theory of mind: Neuropsychological evidence from a false-belief task with reduced language and executive demands. Journal of Cognitive Neuroscience, 16, 1773-1784. doi:10.1162/0898929042947928

Aron, A., Aron, E. N., \& Smollan, D. (1992). Inclusion of Other in the Self Scale and the structure of interpersonal closeness. Journal of Personality and Social Psychology, 63, 596-612.

Aron, A., Aron, E. N., \& Norman, C. (2001). Self expansion model of motivation and cognition in close relationships and beyond. In G. J. O. Fletcher \& M. S. Clark (Eds.), Blackwell HANDBOOK of social psychology (Interpersonal processes, Vol. 2, pp. 478-500). Oxford, UK: Blackwell.

Avlund, K., Kreiner, S., \& Schultz-Larsen, K. (1993). Construct validation and the Rasch model: Functional ability of healthy elderly people. Scandinavian Journal of Public Health, 21, 233-246.

Bowles, R. P., \& Salthouse, T. A. (2003). Assessing the age-related effects of proactive interference on working memory tasks using the Rasch model. Psychology and Aging, 18, 608-615.

Cavallini, E., Bianco, F., Bottiroli, S., Rosi, A., Vecchi, T., \& Lecce, S. (2015). Training for generalization in Theory of Mind: A study with older adults. Frontiers in Psychology, 6, 1123. doi:10.3389/fpsyg. 2015.01123

Conway, M. A., \& Pleydell-Pearce, C. W. (2000). The construction of autobiographical memories in the self-memory system. Psychological Review, 107, 261-288. doi:10.1037/0033-295X. 107.2.261

Cunningham, S. J., Turk, D. J., Macdonald, L. M., \& Macrae, C. N. (2008). Yours or mine? Ownership and memory. Consciousness and Cognition, 17, 312-318. doi:10.1016/j.concog.2007.04.003

Emery, L., Hale, S., \& Myerson, J. (2008). Age differences in proactive interference, working memory, and abstract reasoning. Psychology and Aging, 23, 634-645. doi:10.1037/a0012577

Fredrickson, B. L., \& Carstensen, L. L. (1990). Choosing social partners: How old age and anticipated endings make people more selective. Psychology and Aging, 5, 335-347.

Fung, H. H., Carstensen, L. L., \& Lutz, A. (1999). The influence of time on social preferences: Implications for life-span development. Psychology and Aging, 14, 595-604. doi:10.1037/0882-7974.14.4.595

Gow, A. J., \& Mortensen, E. L. (2016). Social resources and cognitive ageing across 30 years: The Glostrup 1914 Cohort. Age and Ageing, 45, 480-486. doi:10.1093/ageing/afw070

Grady, C. L., \& Craik, F. I. M. (2000). Changes in memory processing with age. Current Opinion in Neurobiology, 10, 224-231. doi:10. 1016/S0959-4388(00)00073-8 
Gutchess, A. H., Kensinger, E. A., Yoon, C., \& Schacter, D. L. (2007). Ageing and the self-reference effect in memory. Memory, 15, 822 837. doi:10.1080/09658210701701394

Gutchess, A. H., Kensinger, A. E., \& Schacter, D. L. (2010). Functional neuroimaging of self-referential encoding with age. Neuropsychologia, 48, 211-219. doi:10.1016/j.neuropsychologia. 2009.09.006

Hamami, A., Serbun, S. J., \& Gutchess, A. H. (2011). Self-referencing enhances memory specificity with age. Psychology and Aging, 26, 636-646. doi:10.1037/a0022626

Henry, J. D., Phillips, L. H., Ruffman, T., \& Bailey, P. E. (2013). A metaanalytic review of age differences in theory of mind. Psychology and Aging, 28, 826-839. doi:10.1037/a0030677

Holm, S. (1979). A simple sequentially rejective multiple test procedure. Scandinavian Journal of Statistics, 6, 65-70.

Humphreys, G. W., \& Sui, J. (2015). The salient self: Social saliency effects based on self bias. Journal of Cognitive Psychology, 27, 129-140. doi:10.1080/20445911.2014.996156

Lakens, D. (2013). Calculating and reporting effect sizes to facilitate cumulative science: A practical primer for $t$ tests and ANOVAs. Frontiers in Psychology, 4, 863. doi:10.3389/fpsyg.2013.00863

Mather, M., \& Carstensen, L. L. (2005). Aging and motivated cognition: The positivity effect in attention and memory. Trends in Cognitive Sciences, 9, 496-502. doi:10.1016/j.tics.2005.08.005

Mueller, J. H., Wonderlich, S., \& Dugan, K. (1986). Self-referent processing of age-specific material. Psychology and Aging, 1, 293-299. doi:10.1037/0882-7974.1.4.293

Rabbitt, R. (1997). Methodology of frontal and executive function. Hove, UK: Psychology Press.

Rosa, N. M., \& Gutchess, A. H. (2011). Source memory for action in young and older adults: Self vs. close or unknown others. Psychology and Aging, 26, 625-630. doi:10.1037/a0022827

Salthouse, T. A. (2009). When does age-related cognitive decline begin? Neurobiology of Aging, 30, 507-514. doi:10.1016/j.neurobiolaging. 2008.09.023

Shamay-Tsoory, S. G., \& Aharon-Peretz, J. (2007). Dissociable prefrontal networks for cognitive and affective theory of mind: A lesion study. Neuropsychologia, 45, 3054-3067. doi:http://dx.doi.org/10.1016/j. neuropsychologia.2007.05.021

Slessor, G., Phillips, L. H., \& Bull, R. (2007). Exploring the specificity of age-related differences in theory of mind tasks. Psychology and Aging, 22, 639-643. doi:10.1037/0882-7974.22.3.639

Slessor, G., Phillips, L. H., \& Bull, R. (2008). Age-related declines in basic social perception: Evidence from tasks assessing eye-gaze processing. Psychology and Aging, 23, 812-822. doi:10.1037/ a0014348
Stolte, M., Humphreys, G. W., Yankouskaya, A., \& Sui, J. (2015). Dissociating biases towards the self and positive emotion. Quarterly Journal of Experimental Psychology, 7, 1-34. doi:10. 1080/17470218.2015.1101477

Sui, J. (2016). Self-reference acts as a golden thread in binding. Trends in Cognitive Sciences, 20, 482-483.

Sui, J., \& Humphreys, G. W. (2014). Super-size me: Self biases increase to larger stimuli. Psychonomic Bulletin \& Review, 22, 550-558. doi: 10.3758/s13423-014-0690-6

Sui, J., \& Humphreys, G. W. (2015a). The integrative self: How selfreference integrates perception and memory. Trends in Cognitive Sciences, 19, 719-728. doi:10.1016/j.tics.2015.08.015

Sui, J., \& Humphreys, G. W. (2015b). The interaction between self-bias and reward: Evidence for common and distinct processes. Quarterly Journal of Experimental Psychology, 68, 1952-1964. doi:10.1073/ pnas. 1221862110

Sui, J., He, X., \& Humphreys, G. W. (2012). Perceptual effects of social salience: Evidence from self-prioritization effects on perceptual matching. Journal of Experimental Psychology: Human Perception and Performance, 38, 1105-1117. doi:10.1037/ a0029792

Sui, J., Rotshtein, P., \& Humphreys, G. W. (2013). Coupling social attention to the self forms a network for personal significance. Proceedings of the National Academy of Sciences, 110, 7607-7612.

Sui, J., Enock, F., Ralph, J., \& Humphreys, G. W. (2015). Dissociating hyper- and hypo-self biases to a core self-representation. Cortex, 70, 202-212. doi:10.1016/j.cortex.2015.04.024

Sun, Y., Fuentes, L. J., Humphreys, G. W., \& Sui, J. (2016). Try to see it my way: Embodied perspective enhances self and friend-biases in perceptual matching. Cognition, 153, 108-117. doi:10.1016/j. cognition.2016.04.015

Symons, C. S., \& Johnson, B. T. (1997). The self-reference effect in memory: A meta-analysis. Psychological Bulletin, 121, 371-394. doi: 10.1037/0033-2909.121.3.371

Trelle, A. N., Henson, R. N., \& Simons, J. S. (2015). Identifying ageinvariant and age-limited mechanisms for enhanced memory performance: Insights from self-referential processing in younger and older adults. Psychology and Aging, 30, 324-333. doi:10.1037/ a0039116

Wimmer, H., \& Perner, J. (1983). Beliefs about beliefs: Representation and constraining function of wrong beliefs in young children's understanding of deception. Cognition, 13, 103-128. doi:10.1016/ 0010-0277(83)90004-5

Yang, L., Truong, L., Fuss, S., \& Bislimovic, S. (2012). The effects of ageing and divided attention on the self-reference effect in emotional memory: Spontaneous or effortful mnemonic benefits. Memory, 20, 596-607. doi:10.1080/09658211.2012.690040 